\title{
Modifications in Nanoparticle-Protein Interactions by varying the Protein Conformation
}

\author{
Sugam Kumar ${ }^{1}$, I. Yadav ${ }^{1}$, V. K. Aswal ${ }^{1 *}$ and J. Kohlbrecher ${ }^{2}$ \\ ${ }^{1}$ Solid State Physics Division, Bhabha Atomic Research Centre, Mumbai 400 085, India \\ ${ }^{2}$ Laboratory for Neutron Scattering, Paul Scherrer Institute, CH-5232 PSI Villigen, Switzerland \\ E-mail: vkaswal@barc.gov.in
}

\begin{abstract}
Small-angle neutron scattering has been used to study the interaction of silica nanoparticle with Bovine Serum Albumin (BSA) protein without and with a protein denaturing agent urea. The measurements have been carried out at $\mathrm{pH} 7$ where both the components (nanoparticle and protein) are similarly charged. We show that the interactions in nanoparticle-protein system can be modified by changing the conformation of protein through the presence of urea. In the absence of urea, the strong electrostatic repulsion between the nanoparticle and protein prevents protein adsorption on nanoparticle surface. This non-adsorption, in turn gives rise to depletion attraction between nanoparticles. However, with addition of urea the depletion attraction is completely suppressed. Urea driven denaturation of protein is utilized to expose the positively charged patched of the BSA molecules which eventually leads to adsorption of BSA on nanoparticles eliminating the depletion interaction.
\end{abstract}

Keywords: Nanoparticle-protein interaction, Protein conformation, Depletion interaction, SANS

PACS: 61.05.fg, 61.46.Df, 82.70.Dd

\section{INTRODUCTION}

In the field of nanobiotechnology, nanoparticleprotein interactions have gained sufficient scientific interest because they can access and operate within the cell because of their size, which is comparable to that of a cell [1]. As a result, the nanoparticle-protein conjugates find enormous applications in targeted drug delivery, protein crystallization, biological imaging, development of biocompatible functional materials, etc. Majority of these applications require understanding of nanoparticle-protein interactions which depend on the intrinsic characteristics (e.g., sizes, shapes, and charges) of both the nanoparticles and proteins [2].

In the suitable physiological environment, proteins are known to adsorb on nanoparticles immediately as they come into the contact, and therefore protein structure and functionality may be disturbed. However, in the case, if the two components (nanoparticle and protein) are having similar charge natures (anionic or cationic), strong electrostatic repulsion prevents them from forming a conjugate and the protein structure remains almost intact. Such non-adsorption of protein molecules can give rise to attractive depletion force between nanoparticles. Recently, we have also reported such Bovine Serum Albumin (BSA) protein induced depletion attraction between silica nanoparticles at $\mathrm{pH} 7$ where both the components are similarly charged [3, 4]. At sufficient concentration of the protein, the enhanced depletion interaction ultimately leads to the nanoparticle aggregation. Herein, we report the complete suppression of the depletion driven nanoparticle aggregation by changing the protein conformation using a well known protein denaturing agent urea. The phase behavior of nanoparticle-protein system is found to be significantly modified in presence of urea. Small-angle neutron scattering (SANS) has been used to investigate the system as it provides information on both structure and interaction in in-situ and under native environment [5].

\section{EXPERIMENT}

Charge stabilized colloidal suspension of silica nanoparticles (Ludox HS40) and Bovine Serum Albumin (M. W. $66.4 \mathrm{kD}$ and I.P. 4.7) protein were purchased from Sigma-Aldrich. Samples for SANS experiments were prepared by dissolving weighted amount of nanoparticle and protein in $20 \mathrm{mM}$ phosphate buffer at $\mathrm{pH} 7$ prepared in $\mathrm{D}_{2} \mathrm{O}$. Small-angle neutron scattering experiments were performed at SANS-I facility, Swiss Spallation Neutron Source SINQ, Paul Scherrer Institute, Switzerland. The 
wavelength $(\lambda)$ of neutron beam used was $0.6 \mathrm{~nm}$ and the scattered neutrons from samples were detected using two-dimensional $\left(96 \times 96 \mathrm{~cm}^{2}\right)$ detector. Data were collected at two sample-to-detector distances 2 and $8 \mathrm{~m}$ to cover a wave vector transfer $(\mathrm{Q}=4 \pi \sin (\theta / 2) / \lambda$, where $\theta$ is scattering angle $)$ range of 0.04 to $2.5 \mathrm{~nm}^{-1}$. The data were corrected and normalized to absolute scale using standard procedure.

\section{SANS ANALYSIS}

In SANS experiments, the coherent differential scattering cross section per unit volume $(\mathrm{d} \Sigma / \mathrm{d} \Omega)$ is measured as a function of $Q$. For a monodisperse particle system in a medium, it may be given by [6]

$$
\frac{d \Sigma}{d \Omega}(Q)=n P(Q) S(Q)+B
$$

where $n$ is number density of particles. $P(Q)$ is intraparticle structure factor (square of the form factor) and $S(Q)$ is interparticle structure factor. $\mathrm{B}$ is a constant term representing incoherent background coming mainly from the hydrogen present in sample.

$\mathrm{P}(\mathrm{Q})$ is decided by the shape and size of the particle. For spherical particle of radius $R$, it may be written as [6]

$$
P(Q)=\frac{16 \pi^{2}}{9}\left(\rho_{p}-\rho_{s}\right)^{2} R^{6}\left[3 \frac{\sin (Q R)-(Q R) \cos (Q R)}{(Q R)^{3}}\right]^{2}
$$

where $\rho_{\mathrm{p}}$ and $\rho_{\mathrm{s}}$ are the scattering length densities of particle and solvent, respectively.

Interparticle structure factor $S(Q)$ depends upon the spatial arrangement of the particles. It may be approximated to unity if the sample is dilute and effectively there is no particle-particle interaction at average distances. But in general, the interaction between the particles may be a combination of attractive and repulsive forces. In this case, $S(Q)$ may be calculated by solving the Ornstein-Zernike (OZ) equation for two-Yukawa (2Y) potential accounting both attraction as well as repulsion under Mean Spherical Approximation (MSA) closure relation [7].

$$
\begin{aligned}
U(r) & =\infty \text { for } 0<r<\sigma \\
& =-K_{1} \frac{\exp \left\{-\alpha_{1}(r / \sigma-1)\right\}}{r / \sigma}+K_{2} \frac{\exp \left\{-\alpha_{2}(r / \sigma-1)\right\}}{r / \sigma} \text { for } r>\sigma
\end{aligned}
$$

where $K$ (in units of $k_{B} T, k_{B}$ is Boltzmann constant and $T$ is temperature) is proportional to magnitude of the potential and $1 / \alpha$ is proportional to the range of the potential. The data have been analyzed by comparing the scattering from different models to the experimental data.

\section{RESULTS AND DISCUSSION}

Fig. 1 shows the SANS data of $1 \mathrm{wt} \%$ HS40 silica nanoparticles and $1 \mathrm{wt} \% \mathrm{BSA}$ protein in $\mathrm{D}_{2} \mathrm{O}$. Both the systems (nanoparticle and protein) show a monotonically decreasing profile as a function of $Q$. At $1 \mathrm{wt} \%$ concentration, the interparticle structure factor $S(Q)$ contribution can be neglected and therefore the scattering is mostly governed by intraparticle structure factor $P(Q)$. Silica nanoparticles are modeled by the polydisperse spheres having radius of $8.0 \mathrm{~nm}$ with polydispersity 0.2 . The BSA is a globular protein and is fitted with oblate ellipsoidal shape. The calculated values of semi-minor $(a)$ and semi-major $(b=c)$ axes are $1.4 \mathrm{~nm}$ and $4.2 \mathrm{~nm}$, respectively.

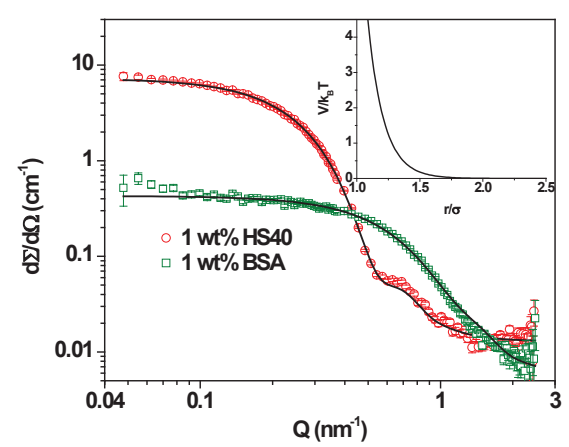

FIGURE 1. SANS data of $1 \mathrm{wt} \%$ HS40 silica nanoparticles and $1 \mathrm{wt} \%$ BSA protein solutions. The inset shows the repulsive interaction potential between nanoparticles calculated from concentrated (10 wt \%) solution [3].

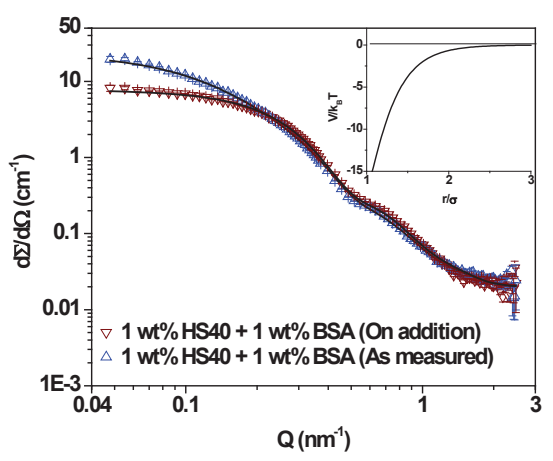

FIGURE 2. SANS data of $1 \mathrm{wt} \%$ HS40 with $1 \mathrm{wt} \%$ BSA. The measured SANS data are compared with that obtained on addition of scattering data from two individual components. The inset shows the calculated total interaction potential between nanoparticles in the presence of BSA.

Fig. 2 shows the SANS data of $1 \mathrm{wt} \%$ HS40 silica nanoparticles with $1 \mathrm{wt} \% \mathrm{BSA}$ protein. It is observed that the data of silica nanoparticles with BSA protein are quite different from addition of scattering data from two individual components. The data of the mixed system show a scattering buildup at the low $Q$ values whereas that at the high $Q$ values is mostly 
governed by scattering from BSA protein. The low $Q$ rise of the scattering intensity suggests evolution of attractive interaction in the system which may be attributed to the protein induced depletion interaction between nanoparticles [3, 4]. The data in this case have been fitted by calculating $S(Q)$ using a two-Yukawa potential accounting for depletion attraction as well as electrostatic repulsion between nanoparticles. The fitted total interaction potential is shown in the inset of the Fig. 2. As can be seen, the total potential becomes attractive instead of repulsive for pure nanoparticle system (inset of Fig. 1).

Fig. 3 shows the effect of urea on nanoparticleprotein system where the SANS data of $1 \mathrm{wt} \% \mathrm{HS} 40+$ $1 \mathrm{wt} \% \mathrm{BSA}+3 \mathrm{M}$ urea have been plotted and compared with that of $1 \mathrm{wt} \% \mathrm{HS} 40+1 \mathrm{wt} \%$ BSA. The data of $1 \mathrm{wt} \% \mathrm{HS} 40+1 \mathrm{wt} \% \mathrm{BSA}+3 \mathrm{M}$ urea are corrected for incoherent background and scaled for contrast. No scattering buildup in the low $\mathrm{Q}$ range is observed with addition of urea suggesting the profound effect of urea on depletion interaction. Interestingly, the data of $1 \mathrm{wt} \% \mathrm{HS} 40+1 \mathrm{wt} \% \mathrm{BSA}+$ $3 \mathrm{M}$ urea system completely overlaps on that of pure nanoparticles.

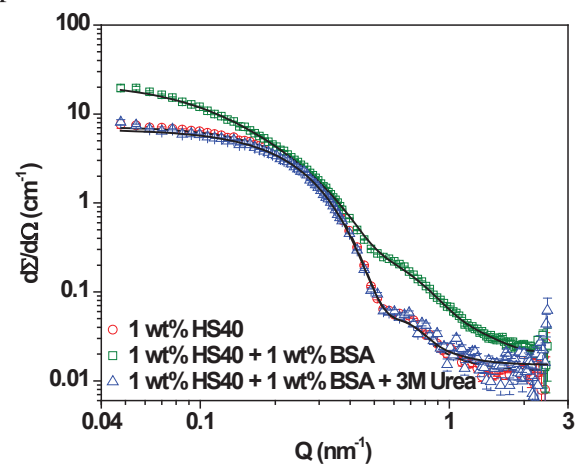

FIGURE 3. SANS data of $1 \mathrm{wt} \%$ HS $40,1 \mathrm{wt} \%$ HS $40+$ $1 \mathrm{wt} \%$ BSA and $1 \mathrm{wt} \%$ HS $40+1 \mathrm{wt} \%$ BSA $+3 \mathrm{M}$ urea systems. The data of $1 \mathrm{wt} \% \mathrm{HS} 40+1 \mathrm{wt} \% \mathrm{BSA}+3 \mathrm{M}$ urea are corrected for background and scaled for comparison.

The urea effect on nanoparticle-protein interaction be explained in terms of urea induced denaturation of protein molecules. Urea driven unfolding of protein is expected to expose the positively charged patched of the BSA molecules. This unfolding of protein molecules is believed to be the driving force for the site-specific weak adsorption of protein molecules on nanoparticles [8], eliminating depletion attraction. However, adsorption remains weak in this case and hence could not be reflected in SANS data. The adsorption increases on increasing urea concentration as more and more protein are unfolded and contribute in the formation of protein corona around nanoparticles. The effect of urea concentration on nanoparticle-protein interactions is depicted in Fig. 4.
The scattering curve becomes narrower with increasing urea concentration which indicates the increase in the size of the scatterer. The increased size of the particles can be attributed to the formation of protein corona around the nanoparticles. The data is fitted with core-shell model with spherical core of nanoparticle and shell of adsorbed protein. The shell thickness is found to be $1.2 \mathrm{~nm}$ which is less than the minor axis $(2 a=2.8 \mathrm{~nm})$ of the protein corroborating the unfolding of the protein.

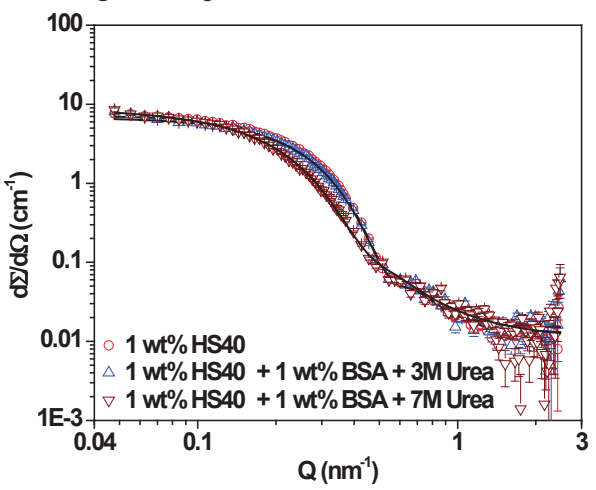

FIGURE 4. SANS data of 1 wt $\%$ HS $40+1$ wt $\%$ BSA with varying concentration of urea.

\section{CONCLUSIONS}

The interaction of silica nanoparticle with BSA protein has been investigated by varying the protein conformation through a denaturing agent urea. The measurements have been performed at $\mathrm{pH} 7$ where both the components are similarly charged. In absence of urea, electrostatic repulsion between the two components prevents protein adsorption on nanoparticle, rather non-adsorbing nature of protein produces depletion force that causes the aggregation of nanoparticles. However, addition of urea completely suppresses the depletion attraction. Urea driven denaturation of protein is used to expose the positively charged patched of the BSA molecules which eventually leads to a weak adsorption of BSA on nanoparticles eliminating the depletion interaction.

\section{REFERENCES}

1. J. Leszczynski, Nature Nanotechnol. 5, 633-634 (2010).

2. I.Lynch and K. A. Dawson, NanoToday 3, 40-47 (2008).

3. I. Yadav, S. Kumar,V. K. Aswal and J. Kohlbrecher, Phys. Rev. E 89, 032304 (2014).

4. I. Yadav, V. K. Aswal and J. Kohlbrecher, Phys. Rev. E 93, 052601 (2016).

5. S. Kumar, V. K. Aswal, and P. Callow, Langmuir 30, 1588-1598 (2014).

6. J. S. Pedersen, Adv. Coll. Inter. Sci. 70, 171-210 (1997).

7. Y. Liu, W.-R. Chen, and S.-H. Chen, J. Chem. Phys. 122, 044507 (2005)

8. J.S. Gebauer, M. Malissek, S. Simon, S.K. Knauer, M. Maskos, R.H. Stauber, W. Peukert and L. Treuel, Langmuir, 28, 9673-9679 (2012). 